\title{
Comprehensive Molecular Characterization of Chinese Patients with Glioma by Extensive Next-Generation Sequencing Panel Analysis
}

\author{
Chun Zeng ${ }^{1,2}$ \\ Jing Wang ${ }^{3}$ \\ Mingwei $\mathrm{Li}^{4}$ \\ Huina Wang ${ }^{4}$ \\ Feng Lou ${ }^{4}$ \\ Shanbo $\mathrm{Cao}^{4}$ \\ Changyu $\mathrm{Lu}^{3}$
}

'Department of Neurosurgery, Beijing Tiantan Hospital, Capital Medical University, Beijing, People's Republic of China; ${ }^{2}$ China National Clinical Research Center for Neurological Diseases, Beijing, People's Republic of China; ${ }^{3}$ Department of Neurosurgery, Peking University International Hospital, Beijing, People's Republic of China; ${ }^{4}$ Acornmed Biotechnology Co., Ltd, Beijing, People's Republic of China
Correspondence: Changyu Lu Department of Neurosurgery, Peking University International Hospital, Beijing, People's Republic of China

$\mathrm{Tel}+8669006 \mid 44$

Email luchangyu2020@I63.com

Shanbo Cao

Acornmed Biotechnology Co., Ltd, Floor

18, Block 5, Yard 18, Kechuang 13 Road,

Beijing, I00I76, People's Republic of

China

Tel +8653606156

Email shanbocao@acornmed.com
Background: Tremendous efforts have been made to explore biomarkers for classifying and grading glioma. However, the majority of the current understanding is based on public databases that might not accurately reflect the Asian population. Here, we investigated the genetic landscape of Chinese glioma patients using a validated multigene next-generation sequencing (NGS) panel to provide a strong rationale for the future classification and prognosis of glioma in this population.

Methods: We analyzed 83 samples, consisting of 71 initial treatments and 12 recurrent surgical tumors, from 81 Chinese patients with gliomas by performing multigene NGS with an Acornmed panel targeting 808 cancer-related hotspot genes, including genes related to glioma (hotspots, selected exons or complete coding sequences) and full-length SNPs located on chromosomes 1 and 19.

Results: A total of $76(91.57 \%)$ glioma samples had at least one somatic mutation. The most commonly mutated genes were TP53, TERT, IDH1, PTEN, ATRX, and EGFR. Approximately one-third of cases exhibited more than one copy number variation. Of note, this study identified the amplification of genes, such as EGFR and PDGFRA, which were significantly associated with glioblastoma but had not been previously used for clinical classification $(\mathrm{P}<0.05)$. Significant differences in genomic profiles between different pathological subtypes and WHO grade were observed. Compared to the MSKCC database primarily comprised of Caucasians, $H 3 F 3 A$ mutations and $M E T$ amplifications exhibited higher mutation rates, whereas TERT mutations and EGFR and $C D K N 2 A / B$ copy number variations presented a lower mutation rate in Chinese patients with glioma $(\mathrm{P}<0.05)$.

Conclusion: Our multigene NGS in the simultaneous evaluation of multiple relevant markers revealed several novel genetic alterations in Chinese patients with glioma. NGSbased molecular analysis is a reliable and effective method for diagnosing brain tumors, assisting clinicians in evaluating additional potential therapeutic options, such as targeted therapy, for glioma patients in different racial/ethnic groups.

Keywords: glioma, molecular pathology, molecular biomarker, copy number variations, multigene NGS panel

\section{Introduction}

Gliomas are the most common tumors in the brain and central nervous system (CNS). ${ }^{1}$ However, gliomas comprise a diverse collection of entities, each with its own unique clinical and biological characteristics. ${ }^{2}$ According to the 2016 World Health Organization (WHO) standards, gliomas include grade II-III astrocytoma 
(A) and oligodendroglioma (O), as well as grade IV glioblastoma (GBM) and diffuse midline glioma (DMG). ${ }^{3}$ Previous brain tumor classification was primarily based on histopathological characteristics confirmed by hematoxylin and eosin staining (H\&E) and immunohistochemistry (IHC). Detection of IDH mutations, $1 \mathrm{p} / 19 \mathrm{q}$ codeletion, and $H 3 F 3 A$ mutations (midline position) are now recommended according to their histologic diagnosis. ${ }^{4-6}$ Emerging evidence has shown that inclusion of molecular characterization could improve the prognostic and predictive stratification of CNS tumors. ${ }^{7-9}$ In fact, many key mutations in the TERT, ATRX, TP53, and EGFR genes found in gliomas ${ }^{10,11}$ could be used as diagnostic, prognostic, and therapeutic biomarkers. However, these mutation markers have not been included in the latest WHO CNS tumor classification criteria; ${ }^{3}$ therefore, they are not used in routine clinical analyses of glioma prognosis or classification. ${ }^{12}$ In addition, malignant gliomas are intrinsic primary CNS tumors, and a recent sequencing analysis reported that these tumors have high intertumoral molecular heterogeneity. ${ }^{13}$ This causes difficulties in the classification and prognostic determinations with implications for targeted therapies in CNS tumors. ${ }^{14}$ Therefore, a comprehensive genetic analysis is urgently needed for precise tumor classification, guiding clinicians in treatment decision-making for this disease.

Targeted next-generation sequencing (NGS) is an effective and practical method because it can rapidly analyze a wide variety of molecular abnormalities in the entire genome, with low DNA input and low cost. ${ }^{15}$ These advantages have made NGS an attractive and efficient molecular platform for the recent classification of gliomas according to the WHO classification. ${ }^{16}$ However, there are several criteria that should be considered when using targeted NGS analysis. The panel should have enough gene numbers to cover a wide range of targets and their various types of genetic mutations, which may have diagnostic, prognostic, predictive, and therapeutic significance for the targeted tumors. The analysis should also have high reliability in formalin-fixed, paraffin-embedded (FFPE) tissues. ${ }^{15,17}$

In this study, we used a validated multigene NGS panel to retrospectively analyze the sequencing results of 83 glioma samples. The panel included multiple genetic tests for simultaneously identifying single nucleotide variants (SNVs), small insertions and deletions, copy number variations (CNVs), splice variants, and gene rearrangements. We identified frequent and novel genetic alterations in Chinese gliomas. These genetic alterations could be explored as a new set of predictive biomarkers for stratifying each glioma subtype and could help clinicians improve the clinical management of glioma in Chinese patients.

\section{Materials and Methods}

\section{Patient Samples}

In this study, a total of 83 samples, including two matched cerebrospinal fluid samples, were obtained from 81 patients recruited by the Department of Neurosurgery, Peking University International Hospital from January 2018 to December 2019. The histological subtypes and grades of all cases were determined by the morphological characteristics of the tumor tissues analyzed by immunohistochemistry (IHC) and the WHO 2016 classification criteria. ${ }^{16}$ The study was conducted in accordance with the Declaration of Helsinki (as revised in 2013). This study was approved by the Medical Ethics Committee of Peking University International Hospital (YN2020QN03), and written informed consent was obtained from all patients.

\section{Genomic DNA Isolation and Targeted NGS}

Next-generation sequencing was performed at AcornMed Biotechnology (Beijing, China) using an Illumina Hiseq4000 platform (Illumina, San Diego, CA, USA). Genomic DNA was isolated from $200 \mu \mathrm{L}$ whole blood with the QIAGEN DNeasy Blood and Tissue Kit (Qiagen, Shanghai, China) according to the manufacturer's instructions. Then, gDNA was sheared before library construction with a Covaris M220 instrument using the recommended settings for 200-bp fragments. The NGS libraries were constructed using the KAPA Hyper Library Preparation Kit (Kapa Biosystems) following the manufacturer's protocol. Hybrid selection was performed with a custom SeqCap EZ Choice Library (Roche NimbleGen). The various libraries were hybridized with the 808 gene panel, which including genes related to glioma (hotspots, selected exons, or complete coding sequences) and fulllength SNPs located on chromosomes 1 and 19. NimbleGen SeqCap EZ Choice was used according to the manufacturer's protocol with modifications. Following hybrid selection, the captured DNA fragments were amplified with 12 cycles of PCR using $1 \times$ KAPA HiFi Hot Start Ready Mix and $2 \mu \mathrm{M}$ Illumina backbone oligonucleotides in $50-\mu \mathrm{L}$ reactions. Library concentration 
was assessed by Fluorometer (Qubit 4.0) and qPCR (KAPA Biosystems), Fragment length was determined on a 2100 Bioanalyzer using the DNA 1000 Kit (Agilent). Finally, the library was sequenced on one lane using 150 paired-end $(2 * 150 \mathrm{bp})$ strategies.

To ensure the quality of data, the following criteria were used to filter raw variant results: average effective sequencing depth on target per sample $\geq 300 \times$; allele mutation frequency $\geq 10 \%$ for single nucleotide variation and insertion or deletion; all reads were filtered by high mapping quality $(\geq 30)$ and base quality $(\geq 30)$; and the mutant reads needed to be supported by positive and negative strands.

\section{NGS Data Analysis}

Raw data recognition, analysis, and feedback were implemented by an automatic analysis and monitoring system. Quality control statistics and preprocessing of raw sequence data were performed by using an in-house QC tool. Burrows-Wheeler Aligner software (BWA, version 0.5.9) was used to align reads to the hg19 version of the human reference genome. PCR duplicates were marked using the MarkDuplicates tool in Picard. We used IndelRealigner and BaseRecalibrator on Genome Analysis Toolkit (GATK; version 3.8) for realignment and recalibration of the BWA alignment results, respectively. The final alignment results were used for variant calling after multialign reads were filtered from the alignment results. The HaplotypeCaller on Genome Analysis Toolkit (GATK; version 3.8) was used to identify variant calling of SNPs and INDELs. All variants were annotated using Annovar. This analysis focused on targetable genetic alterations annotated by categories of evidence Level 1-3 and Level R1 in OncoKB (Memorial Sloan Kettering Cancer Center, New York, NY, http://oncokb.org/). Synonymous mutations are detected and maintained in database but not clinically reported.

\section{Copy Number Variation Analysis}

$\mathrm{CNV}$ analysis was performed using CONTRA (version 2.1.0), ${ }^{18}$ which indicated CNV gain or loss for genes within the panel coverage. The software computes regions per read and calculates the likelihood possibility based on dispersion measurements and coverage ratios. The hidden Markov model was then used to calculate a $\mathrm{CNV}$ classification. The ratios for each region given were 3.5 for copy number gain and 0.5 for copy number loss. LOHs were detected using Control-FREEC (version 11.5) ${ }^{19}$ based on the pileup files and the known human
SNP information (dbSNP142 from UCSC). The $1 \mathrm{p} / 19 \mathrm{q}$ codeletion status was predicted using CollectReadCounts, CollectAllelicCounts, DenoiseReadCounts, and ModelSegments of GATK4. We defined ratio thresholds of less than 0.9 as deletions.

\section{Methylguanine-DNA Methyltransferase (MGMT) Methylation Detection}

MGMT promoter methylation status was determined by methylation-specific PCR (MSP). In total, $2 \mu \mathrm{g}$ of DNA was subjected to bisulfite treatment using the EpiTect Bisulfite kit (Qiagen, Cat. 59104). DNA was cleaned following the manufacturer's instructions and quantified. In all, $30 \mathrm{ng}$ of DNA per sample was PCR-amplified with Platinum SuperFi DNA polymerase (Invitrogen, Cat. 12351-010) and specific primers to detect methylated and unmethylated MGMT promoters. The PCR amplification protocol was as follows: $94^{\circ} \mathrm{C}$ for $1 \mathrm{~min}$, denaturation at $94^{\circ} \mathrm{C}$ for $30 \mathrm{~s}$, annealing at $60^{\circ} \mathrm{C}$ for $30 \mathrm{~s}$, and extension at $70^{\circ} \mathrm{C}$ for $30 \mathrm{~s}$ for 35 cycles, followed by a 7 -min final extension.

\section{Statistical Analysis}

Statistical analysis was performed using GraphPad Prism (Version 7.01, La Jolla, CA, USA). An unpaired $t$-test was used to assess differences between continuous variables. Fisher's exact test or $\chi^{2}$ test was used to analyze clinical features and genetic features between different groups. Spearman's rank correlation coefficient was used to assess the correlation between variables. $P<0.05$ was considered to indicate statistical significance.

\section{Results \\ Patient Characteristics}

A total of 83 glioma samples from 81 patients were collected in this study, two of which were matched cerebrospinal fluid samples, including 71 initial treatment and 12 recurrent surgical tumors. Median patient age was 46 years (5-75 years). Fifty-nine patients were males $(72.84 \%)$, and 22 were females (27.16\%). The analysis included 56 glioma samples with high-grade glioma (HGG): 32 cases of GBM (38.55\%), 8 cases of DMG (9.64\%), 10 cases of anaplastic astrocytoma (AA) (12.05\%), and 6 cases of anaplastic oligodendroglioma (AO) (7.23\%). Twentyseven glioma samples were low-grade glioma (LGG): 10 cases of A (12.05\%), 11 cases of O (13.25\%), 5 cases of pilocytic astrocytoma (PA) $(6.02 \%)$, and one other case 
Table I Clinical Characteristics of 8 I Patients with Glioma

\begin{tabular}{|c|c|}
\hline Patient Characteristics $(\mathbf{N}=\mathbf{8} I)$ & Number (\%) \\
\hline \multicolumn{2}{|l|}{ Gender, n (\%) } \\
\hline Male & 59 (72.84\%) \\
\hline Female & $22(27.16 \%)$ \\
\hline \multicolumn{2}{|l|}{ Age(year), y (range) } \\
\hline Median & 46 \\
\hline Range & $5-75$ \\
\hline$\leq 40$ & $29(35.80 \%)$ \\
\hline$>40$ & $52(64.20 \%)$ \\
\hline \multicolumn{2}{|l|}{ Tumor characteristics $(\mathrm{N}=83)$} \\
\hline \multicolumn{2}{|l|}{ WHO, n (\%) } \\
\hline I & $6(7.23 \%)$ \\
\hline II & $21(25.30 \%)$ \\
\hline III & $15(18.07 \%)$ \\
\hline IV & $4 \mathrm{l}(49.40 \%)$ \\
\hline \multicolumn{2}{|l|}{ Pathology, n (\%) } \\
\hline PA & $5(6.02 \%)$ \\
\hline A & $10(12.05 \%)$ \\
\hline O & II (I3.25\%) \\
\hline AA & $10(12.05 \%)$ \\
\hline $\mathrm{AO}$ & $6(7.23 \%)$ \\
\hline GBM & $32(38.55 \%)$ \\
\hline DMG & $8(9.64 \%)$ \\
\hline Other & $\mathrm{I}(\mathrm{I} .20 \%)$ \\
\hline \multicolumn{2}{|l|}{ Disease status, $\mathrm{n}(\%)$} \\
\hline Primary & $12(14.46 \%)$ \\
\hline Recurrent & $71(85.54 \%)$ \\
\hline \multicolumn{2}{|l|}{ MGMT methylation, $\mathrm{n}(\%)$} \\
\hline Positive & $43(51.81 \%)$ \\
\hline Negative & 39 (46.99\%) \\
\hline NA & I (I.20\%) \\
\hline \multicolumn{2}{|l|}{$\mathrm{Ip} / \mathrm{I} 9 \mathrm{q}$ codeletion } \\
\hline Positive & $10(12.05 \%)$ \\
\hline Negative & $72(86.75 \%)$ \\
\hline NA & $\mathrm{I}(1.20 \%)$ \\
\hline
\end{tabular}

Note: Oligodendrogliomas were identified by their oligodendrocyte components. Abbreviations: PA, pilocytic astrocytoma (WHO grade I); $\mathrm{A}$, astrocytoma (WHO grade II); O, oligodendroglioma (WHO grade II); AA, anaplastic astrocytoma (WHO grade III); AO, anaplastic oligodendroglioma (WHO grade III); GBM, glioblastoma multiforme (WHO grade IV); DMG, diffuse midline glioma.

(1.20\%). Clinical characteristics of the samples are summarized in Table 1.

\section{Comparing the Genomic Profile of the Chinese Cohort to the MSKCC Database}

In this study, genomic analysis was performed using targeted sequencing based on hybrid capture. Of the 83 samples in our cohort, $76(91.57 \%)$ had at least one somatic mutation or $\mathrm{CNV}$, and the median number of mutations per patient was 4 (range $0-45$ ). A total of 401 nonsynonymous somatic mutations were identified in 148 genes. Similar to previous reports, ${ }^{20}$ the most frequently mutated genes in our cohort of gliomas included TP53, TERT, IDH1/2, PTEN, ATRX, and EGFR (Figure 1). Notably, all IDHI mutations replaced the arginine residue on codon 132, all of which were $R 132 H(25 / 25)$ (Figure 1). The frequency of $H 3 F 3 A$ gene mutations in this study was relatively high (9.64\%) (Figure 1). MGMT methylation was positive in $51.81 \%(43 / 83)$, and $1 \mathrm{p} 19 \mathrm{q}$ codeletion was positive in $12.05 \%(10 / 83)$ (Figure 1). MGMT methylation was found in almost all patients with IDHI mutations $(\mathrm{P}<0.0001)$ (Figure 1). Of the 83 samples, 7 simultaneously had $I D H$ mutations, MGMT methylation, and 1p/19q codeletion (Figure 1). Regarding CNVs, EGFR, PDGFRA, MET, KIT, CDK4 amplification, and CDKN2A deletion were common events (Figure 1). In addition, there was a case of YAP1-PARN fusion (Figure 1).

When we compared the 25 most frequently mutated genes found in this study to the Memorial Sloan Kettering Cancer Center database (MSKCC, Clin Cancer Res 2019, 923 glioma patients, which primarily includes Caucasian patients), ${ }^{21}$ we found that the most commonly mutated genes were TP53, TERT, and IDH1, followed by PTEN, ATRX, EGFR, NF1, and H3F3A (Figure 2). There was no significant difference in the frequency of TP53, IDH1, PTEN, ATRX, EGFR, or CIC gene mutations between our cohort of glioma patients and the MSKCC database (Figure 2). However, we found that TERT and $H 3 F 3 A$ exhibited a higher mutation frequency in our cohort, whereas $M E T$ and $C D K N 2 A$ presented a lower mutation frequency in our cohort of glioma patients (Figure 2). These results suggest that there is a difference in genes between Chinese and Caucasian racial backgrounds with respect to glioma.

\section{Concurrent and Exclusive Gene Analysis}

Previous studies using genomic landscape studies have reported multiple cooccurring or mutually exclusive mutations of genes in different cancer types. ${ }^{22,23}$ For example, the coexistence of PDGFRA and EGFR gene amplifications is commonly found in glioblastoma (GBM). ${ }^{24}$ In addition, EGFR amplification and IDHI mutations are mutually exclusive in low-grade gliomas. ${ }^{25}$ Similarly, our study showed that the mutual exclusion and coexistence of these gene mutations occurred in at least five samples (Figure 3). However, 


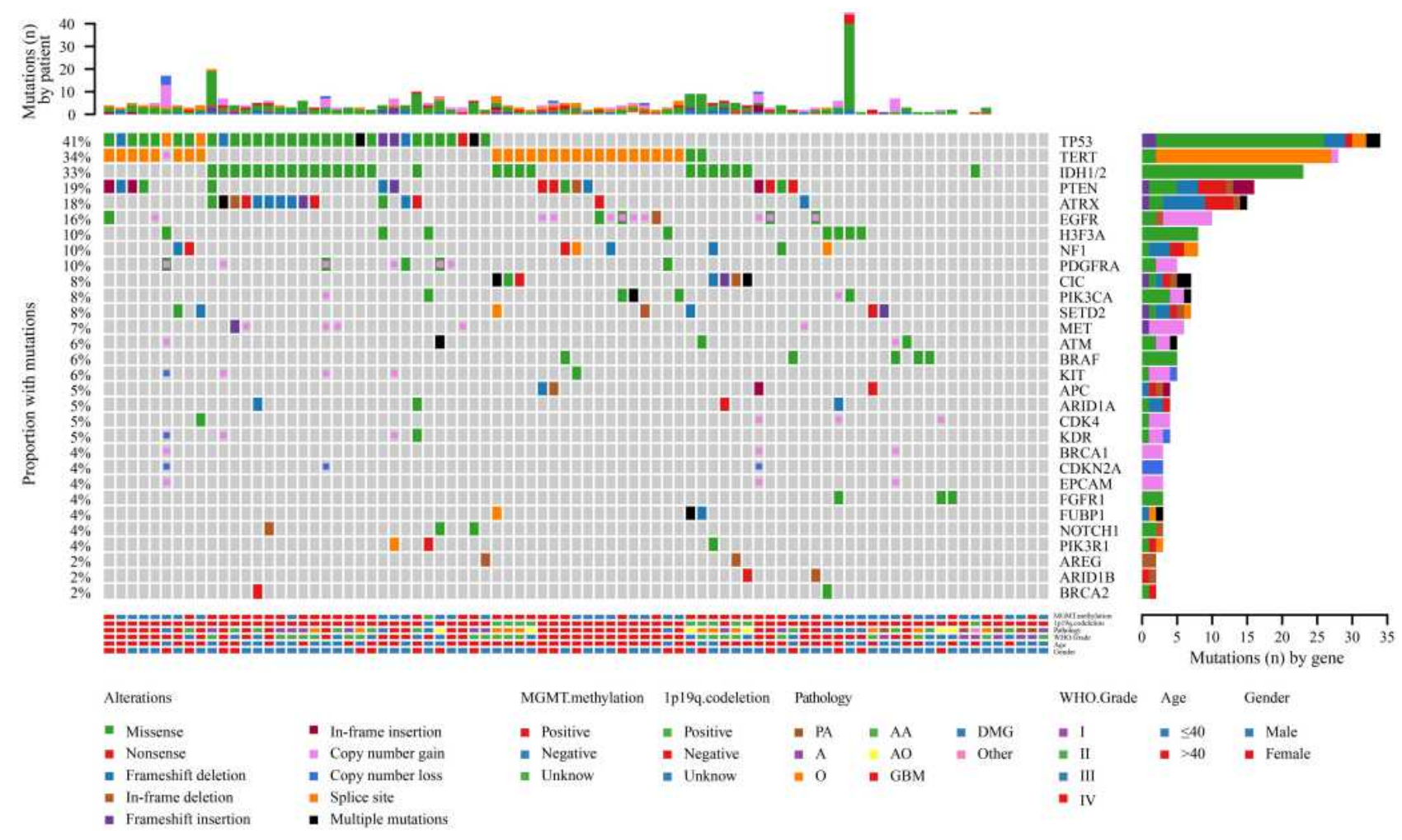

Figure I The landscape of genetic alterations in 75 of 83 samples $(90.36 \%)$ from 8 I glioma cases. Genetic mutations were identified by targeted next-generation sequencing in the tumor tissues of patients. The upper panel shows the numbers of nonsynonymous single-nucleotide variants, small insertions or deletions and copy number variants in each tumor. The heat map below shows genes with somatic mutations sorted according to the mutation frequency. Mutations ( $n$ ) is the number of mutations per gene.

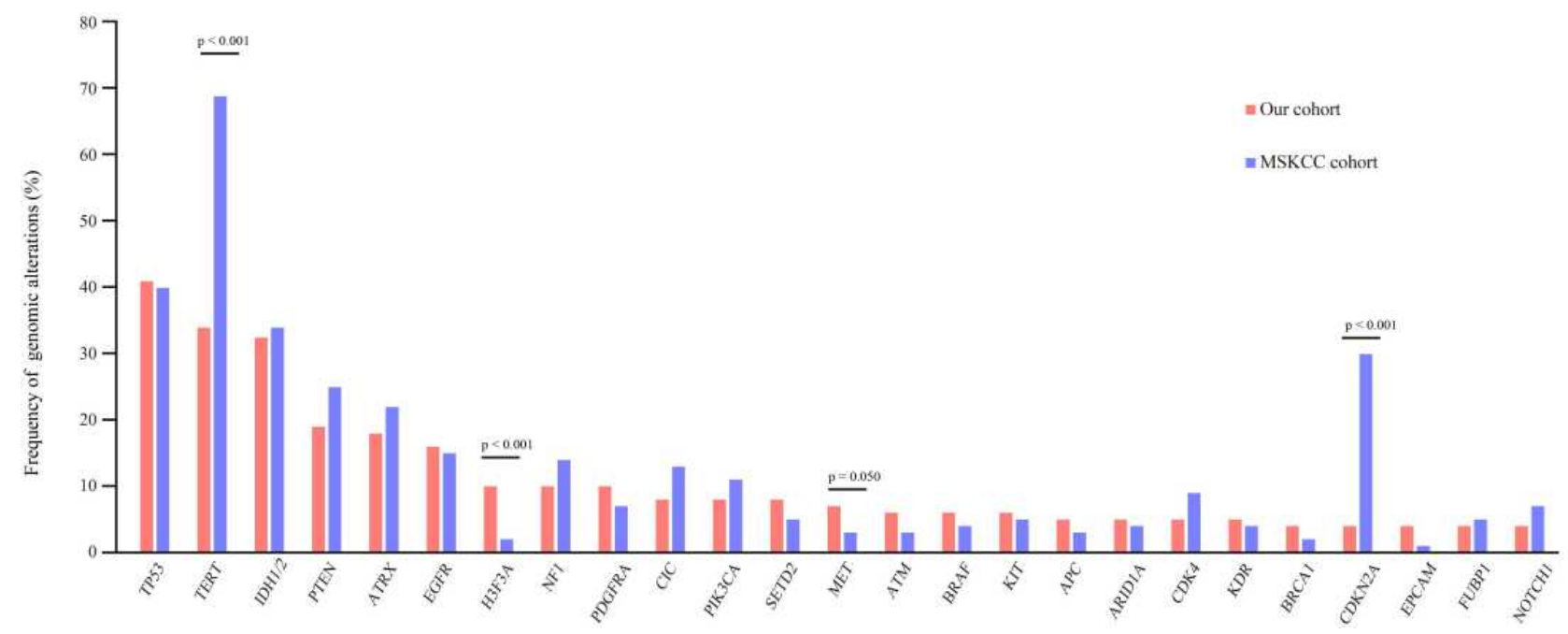

Figure 2 Comparison of mutation frequencies of the top 25 genes from the Chinese and MSKCC cohorts. Commonly mutated genes are arranged in order on the horizontal axis. The vertical axis represents the mutation frequency obtained from a different cohort.

our analysis showed that additional genetic alterations coexisting in gliomas, such as $M G M T$ methylation and IDH1, ATRX, TP53, and CIC somatic mutations, significantly coexisted (Figure 3 ). Other considerable coexisting genes included ATRX and TP53, PTEN and APC, CIC and $M G M T$ methylation, and $1 \mathrm{p} / 19 \mathrm{q}$ codeletion, as well as FUBP1 and TERT and SETD2 $(\mathrm{P}<0.05)$ (Figure 3). In contrast, mutual gene exclusion was relatively rare in 


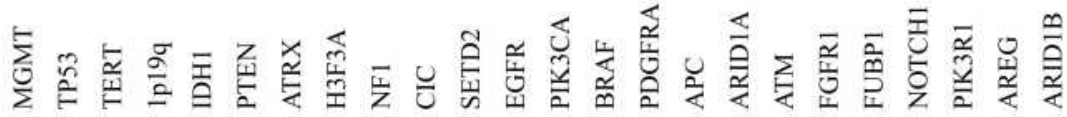

BRCA2

ARIDIB

AREG

PIK3R I

NOTCH1

FUBP1

FGFR I

ATM

ARIDIA

PDGFR

PDGFRA

PIK3CA

EGFR

SETD2

CIC

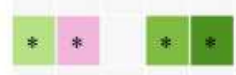

NF1

H3F3A

ATRX

PTEN

IDH1

$1 \mathrm{p} 19 \mathrm{q}$

${ }^{*} \mathbf{p}<0.05$

TERT

TP53

Figure 3 Concurrent and mutually exclusive somatic mutation patterns of significantly mutated genes. Significance was calculated using Fisher's exact test, ${ }^{*} \mathrm{p}<0.05$.

gliomas. Only CIC and TP53, TERT and ATRX, MGMT methylation and $H 3 F 3 A$ showed significant mutual exclusion (Figure 3).

\section{Copy Number Variation (CNV) Analysis}

Copy number variation is a prevalent form of abnormal change in the copy number of several specific genes that commonly occur in tumor development and progression and results in altered gene expression. ${ }^{26,27}$ In this study, NGS-based CNV analysis was used to examine the impact of CNV in glioma in a Chinese cohort. A total of $61 \mathrm{CNVs}$ were identified in 26 samples (31\%), with an average of 2.35 CNVs (1-14) per sample (Figure 4A). There were 53 copy number amplifications and copy number deletions (Figure 4A). CNVs frequently occurred in the age group older than 40 years $(17,20 \%)$ (Figure $4 \mathrm{~A})$. In addition, high-grade gliomas, such as GBM, were characterized by more frequent CNVs $(18,22 \%)$, while low-grade gliomas rarely exhibited $\mathrm{CNV}(\mathrm{P}=0.0001)$, suggesting that $\mathrm{CNV}$ is closely related to patient survival (Table 2).

Previous studies have shown that CNV involves a set of tumor suppressor genes and oncogenes found in GBM. $^{28,29}$ Importantly, these studies showed that GBM with EGFR amplification was significantly associated with both worse disease-free survival (DFS) and overall survival (OS) rates. ${ }^{28,29}$ Hence, the CNV profile in glioma is worth noting in our cohort. In comparison to the 13 genes with the highest $\mathrm{CNV}$ in the MSKCC cohort, we found that the frequencies of CNV in MET, EPCAM, ATM, $C C N E 1, C D K 6$, and $E R B B 2$ were much higher, while $E G F R, C D K 4$, and $C D K N 2 A$ were much lower in our cohort (Figure 4B). However, the frequencies of $\mathrm{CNV}$ in 
A
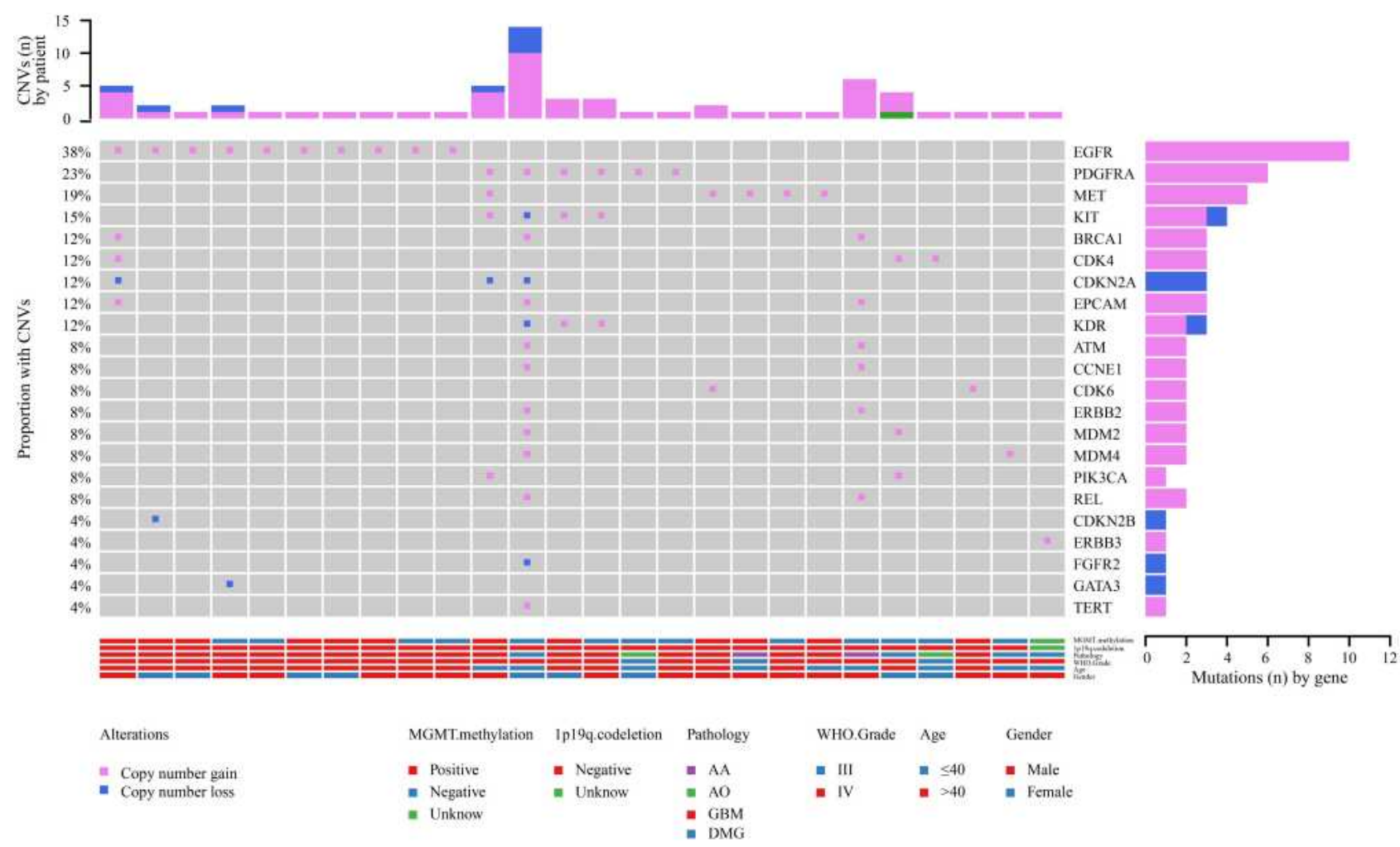

B

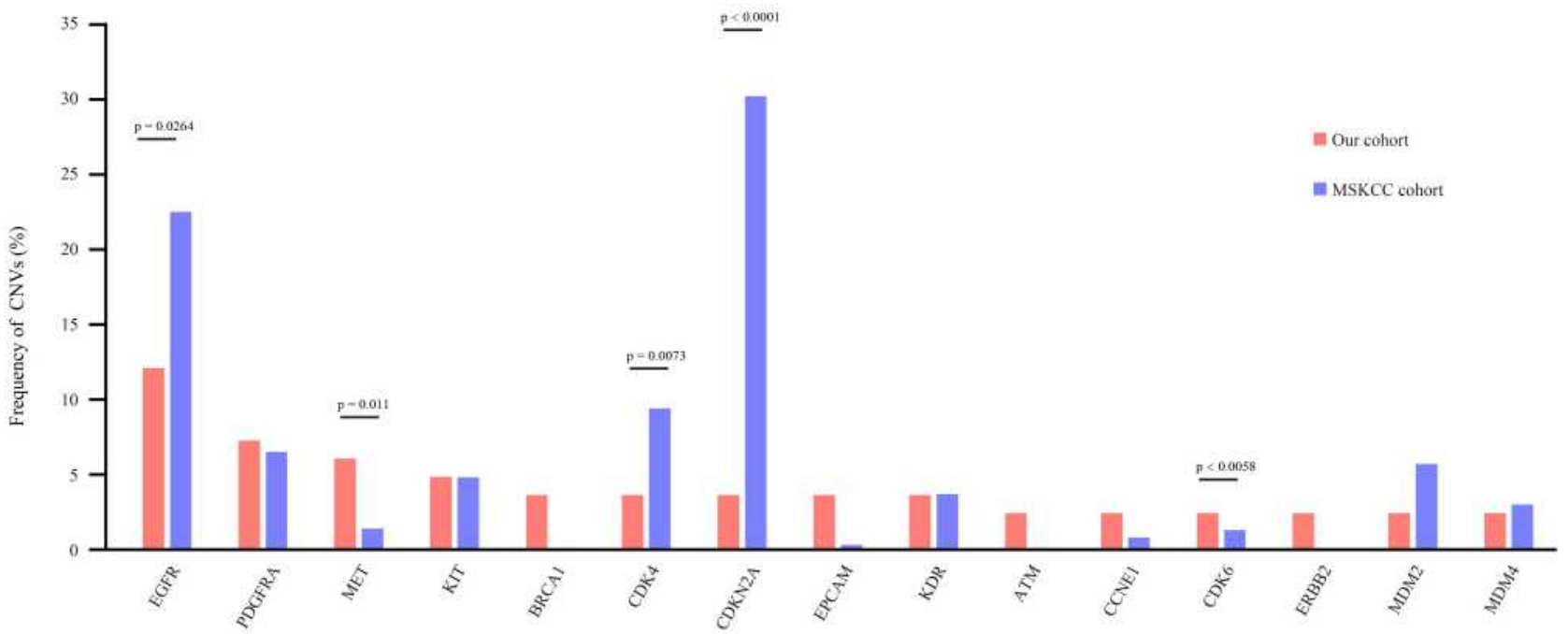

Figure 4 Copy number variation (CNV) analysis. (A) Distribution shift of CNVs in 26 of 83 glioma samples (31.33\%). Copy number losses (blue) and gains (pink) were determined from sequencing data. CNVs $(n)$ is the number of mutations per gene. (B) Comparison of mutation frequencies of CNVs among the top 15 genes from the Chinese and MSKCC cohorts. The commonly CNV genes are arranged in order on the horizontal axis. The vertical axis represents the CNV frequency obtained from a different cohort. 
Table 2 Distributions of Representative Genomic Alterations and Clinical Characteristics Between the Different Pathological Subtypes with Glioma

\begin{tabular}{|c|c|c|c|c|c|c|}
\hline Variable & PA $(N=6)$ & A/AA $(N=20)$ & O/AO $(N=17)$ & GBM $(\mathrm{N}=32)$ & DMG $(\mathbf{N}=8)$ & $\mathbf{P}$ \\
\hline TP53 & 0 & 13 & 3 & 15 & 3 & 0.0101 \\
\hline TERT & 0 & I & 6 & 19 & 2 & 0.0005 \\
\hline IDHI/2 & 0 & 11 & 12 & 4 & 0 & $<0.0001$ \\
\hline PTEN & 0 & 3 & 0 & 12 & I & 0.0123 \\
\hline EGFR & 0 & 0 & 0 & 13 & 0 & $<0.0001$ \\
\hline ATRX & 0 & 7 & 1 & 6 & I & 0.1318 \\
\hline H3F3A & 0 & 0 & 0 & 0 & 8 & $<0.0001$ \\
\hline NFI & 0 & 2 & 1 & 4 & I & 0.8623 \\
\hline PDGFRA & 0 & 0 & I & 5 & 2 & 0.1627 \\
\hline $\mathrm{CIC}$ & 0 & I & 6 & 0 & 0 & 0.0004 \\
\hline PIK3CA & 0 & 0 & 0 & 4 & 3 & 0.0095 \\
\hline SETD2 & 1 & 0 & 3 & 3 & 0 & 0.2862 \\
\hline MET & 0 & 2 & 0 & 4 & 0 & 0.41 \\
\hline ATM & 0 & I & 2 & I & I & 0.6484 \\
\hline BRAF & 0 & 3 & 1 & I & 0 & 0.3771 \\
\hline KIT & 0 & 0 & 0 & 4 & I & 0.2211 \\
\hline$A P C$ & 0 & 0 & 1 & 3 & 0 & 0.5231 \\
\hline ARIDIA & 0 & 3 & 0 & 0 & I & 0.0801 \\
\hline CDK4 & 0 & 0 & 1 & 2 & I & 0.6393 \\
\hline$K D R$ & 0 & 1 & 0 & 2 & 1 & 0.6739 \\
\hline BRCAI & 0 & 1 & 0 & 1 & 1 & 0.5905 \\
\hline CDKN2A & 0 & 0 & 0 & 2 & I & 0.3975 \\
\hline EPCAM & 0 & 1 & 0 & I & I & 0.5905 \\
\hline FGFRI & 0 & 0 & 2 & 0 & 1 & 0.1242 \\
\hline FUBPI & 0 & 0 & 3 & 0 & 0 & 0.0167 \\
\hline NOTCHI & 0 & I & 1 & I & 0 & 0.9234 \\
\hline MGMT & 2 & 13 & 13 & 15 & 0 & 0.0042 \\
\hline $1 p 19 q$ & 0 & 0 & 10 & 0 & 0 & $<0.0001$ \\
\hline CNV & 0 & 2 & 2 & 18 & 4 & 0.0004 \\
\hline Age $\leq 40$ & 5 & 9 & 7 & 3 & 5 & 0.0007 \\
\hline Age $>40$ & 1 & 11 & 10 & 29 & 3 & \\
\hline Male & 6 & 14 & 13 & 23 & 4 & 0.344 \\
\hline Female & 0 & 6 & 4 & 9 & 4 & \\
\hline
\end{tabular}

Note: CNV, the number of glioma patients with CNV events.

PDGFRA, KIT, KDR, and MDM2/4 were not significantly different from those in the MSKCC cohort (Figure 4B).

\section{Mutational Landscape in Pathological Subtypes}

There were fewer gene mutations in PA, which were primarily found in males aged $\leq 40$ years and were under WHO grade I (Table 2). Those $>40$ years old $(\mathrm{P}=0.007)$ were the majority in GBM patients compared to PA, A, $\mathrm{AA}, \mathrm{O}, \mathrm{AO}$, and DMG (Figure 5) (Table 2). Similarly, mutations in TP53 ( $\mathrm{P}=0.0101)$, TERT $(\mathrm{P}=0.0005)$, IDH1 $(\mathrm{P}<0.0001), \quad$ PIK3CA $\quad(\mathrm{P}=0.0095), \quad$ PTEN $\quad(\mathrm{P}=0.0123)$,
EGFR amplification $(\mathrm{P}<0.0001)$, MGMT methylation $(\mathrm{P}=0.0042)$, and $\mathrm{CNV}(\mathrm{P}<0.0001)$ were dominant in GBM compared to the other subtypes (Figure 5) (Table 2). In addition, GBM also exhibited recurring genetic mutations, including ATRX mutations $(6 / 32,19 \%)$ and PDGFRA amplification (5/32, 16\%) (Figure 5) (Table 2). Hence, as expected, among patients with grade II-III A/ AA, TP53 mutation was the most common genomic alteration $(13 / 20,65 \%)$, followed by $I D H 1 / 2$ mutation (11/20, $55 \%)$ and $\operatorname{ATRX}(7 / 20,35 \%)$ (Table 2). Moreover, in the Grade II-III O/AO subgroup, IDH1/2 mutation (12/17, $71 \%)$, TERT mutation $(6 / 17,35 \%)$, and $1 \mathrm{p} 19 \mathrm{q}$ codeletion $(10 / 17,59 \%)$ were the major genetic alterations (Figure 5) 


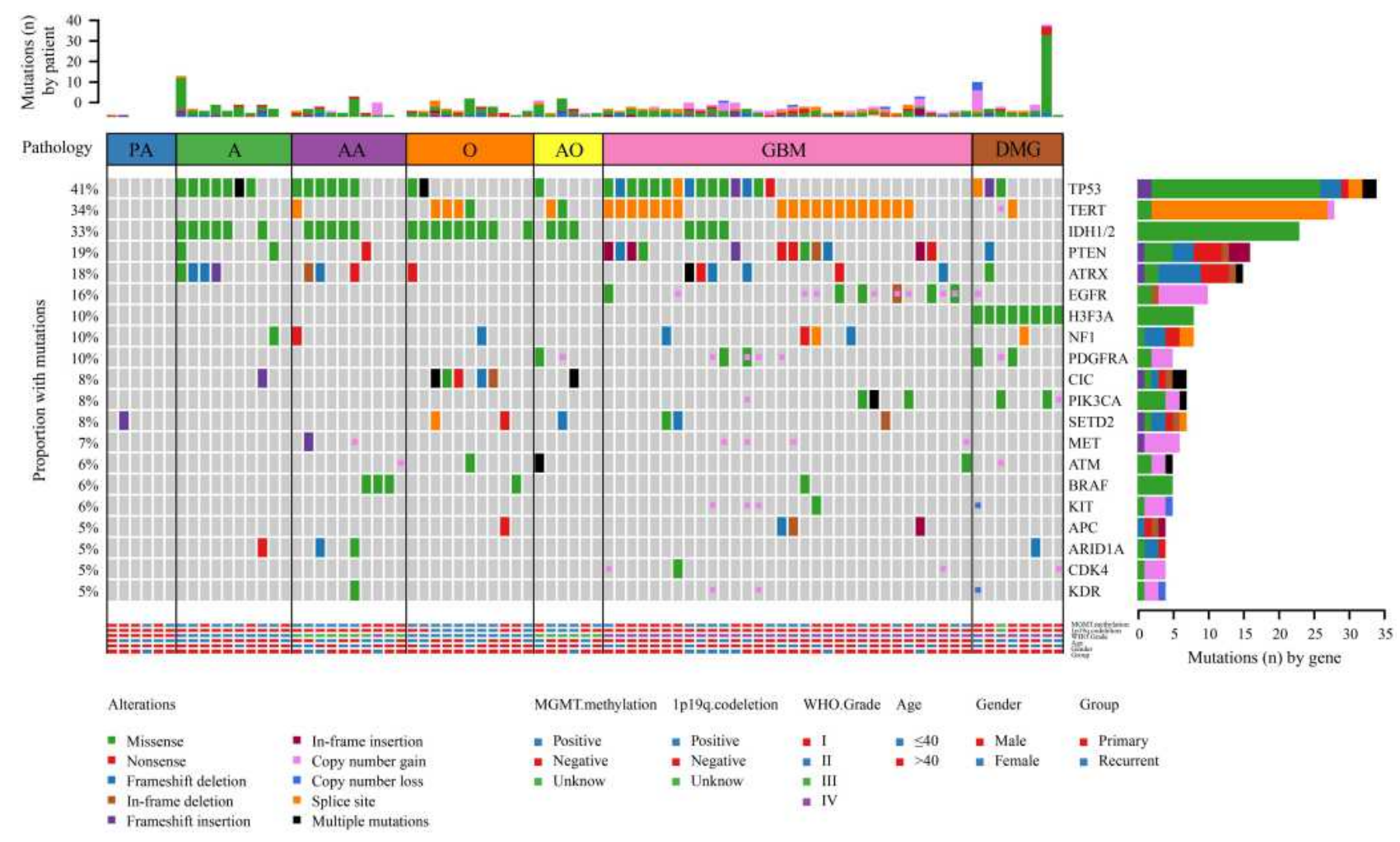

Figure 5 Comprehensive molecular profiles of different pathological subtypes.

(Table 2). Most O/AOs exhibited TERT promoter (6/17, $35 \%), C I C(6 / 17,35 \%)$, and FUBPI (3/17, 18\%) mutations (Table 2). The H3K28M mutation only occurred in DMG patients. Other common genetic alterations in DMG included TP53 mutation (3/8, 38\%), TERT promoter mutation $(2 / 8,25 \%), P D G F R A$ amplification $(2 / 8,25 \%), P T E N$ mutation $(1 / 8,13 \%)$, and $A T R X$ mutation $(1 / 8,13 \%)$, but no mutations were observed in $I D H, E G F R$, or MGMT methylation (Table 2). In addition, MGMT methylation was evenly distributed among the three major subtypes: A/AA (13/20), O/AO (13/17), and GBM (15/32) (Figure 5) (Table 2). These findings indicate that each subtype of glioma has a distinct pattern of genetic alterations, and only multigene NGS analysis is able to identify these gene markers simultaneously.

Our results found that among 17 patients with oligodendroglial tumors only 10 patients with $I D H$ mutations and $1 \mathrm{p} 19 \mathrm{q}$ codeletion. The molecular classification results of 7 patients were inconsistent with the pathological classification results. Two of these cases harbored an IDHI $R 132 \mathrm{H}$ mutation, so the status of $1 \mathrm{p} / 19 \mathrm{q}$ was of additional diagnostic import. The two cases were classified as based on morphology but harbored $1 \mathrm{p} 19 \mathrm{q}$-intacted and TP53 mutation, which is sufficient for the revised diagnosis of
IDH mutation type A (2016 WHO CNS criteria). Five cases harbored $I D H$-wild mutation and $1 \mathrm{p} 19 \mathrm{q}$-intacted, but two cases of them mutated in FGFR1, one case mutated in BRAF V600E, and two cases mutated in other mutations (eg, ATM, PDGFRA), which is for the revised diagnosis of $F G F R$ mutation type, $B R A F$ mutation type and NEC diffuse glioma (cIMPACT-NOW update4). ${ }^{30}$ Here we have demonstrated the utility of NGS panel in identifying the relevant alterations necessary for subtyping $\mathrm{O} / \mathrm{AO}$ using the updated glioma classification.

\section{Analysis of Genomic Features in Different WHO Grades}

In this study, targeted sequencing was used to analyze the pattern of mutations frequently associated with high-grade and low-grade gliomas. We evaluated SNVs, short insertions, deletions, and CNVs of 28 genes in 27 low-grade gliomas (LGGs) and 56 high-grade gliomas (HGGs). There was a significant difference between low-grade and high-grade gliomas with respect to sex and age (Figure 6) (Table 3). Patients $\leq 40$ years old were often found to have low-grade gliomas $(\mathrm{P}=0.0248)$, while women were more likely to develop high-grade gliomas $(\mathrm{P}=0.019)$. We found that mutations in $I D H 1 / 2(\mathrm{P}=0.0019)$ 
and $C I C(\mathrm{P}=0.0017)$ were more prevalent in low-grade gliomas (Figure 6) (Table 3). On the other hand, mutations in TERT $(\mathrm{P}=0.0398), \quad$ EGFR $(\mathrm{P}=0.0064), \quad H 3 F 3 A$ $(\mathrm{P}=0.0388)$, PDGFRA $(\mathrm{P}=0.0388)$, and $\mathrm{CNV}(\mathrm{P}<0.0001)$ were primarily found in high-grade (III-IV) gliomas (Figure 6) (Table 3). Seven gene mutations (TERT, IDH1, EGFR, H3F3A, PDGFRA, and CIC) and CNV were typically observed in HGG (Figure 6) (Table 3). Of note, several genomic mutations have been found only in high-grade tumors, including EGFR and $M E T$ amplification, PTEN genomic deletion, and PIK3CA mutations, indicating that mutations in these genes may represent biomarkers for predicting poor prognosis in high-grade gliomas.

Although high frequencies of $I D H 1 / 2$ mutations are typically used for the molecular genetic classification of gliomas according to the modern WHO CNS tumor classification, ${ }^{3,25}$ our findings indicated that the mutational frequency of IDH1/2 in LGG (15/27) and HGG (12/56) did not provide high accuracy in the classification for grading gliomas (Table 3). On the other hand, previous reports have documented that amplification of chromosome 7 and deletion of chromosome 10 are the primary tumor-driving events in low-grade gliomas. ${ }^{31}$ Consistently, our targeted sequencing results demonstrated that the EGFR gene, which is located on chromosome 7, was amplified in 13/27 LGGs, and the ATRX gene, which is located on chromosome 10 , was deleted in $5 / 27$ LGGs, while there was no detection of either gene in HGGs (Table 3). This suggests that WHO reclassification should consider not only IDH1/IDH2 mutations ${ }^{3}$ but also EGFR amplification and ATRX deletion simultaneously for the diagnosis of glioma tumor grading.

\section{Analysis of Mutated Genes by Different Disease States}

The significant differences in mutated genes between the initial treatment and recurrent groups included $I D H 1 / 2$, $A T R X, C I C, M E T$, and KIT genes. IDH1/2 $(\mathrm{P}=0.0391)$, $A T R X \quad(\mathrm{P}=0.0365), \quad C I C \quad(\mathrm{P}<0.0001), \operatorname{MET}(\mathrm{P}=0.0367)$, and $\operatorname{KIT}(\mathrm{P}=0.0201)$ mutations were significantly higher in recurrent gliomas than in gliomas receiving initial treatment (Figure 7) (Table 4). In addition, the frequency of CNV was 2-fold higher in the recurrence group than in the initial treatment group (Figure 7) (Table 4). However, additional data with a larger sample size are needed for further confirmation.

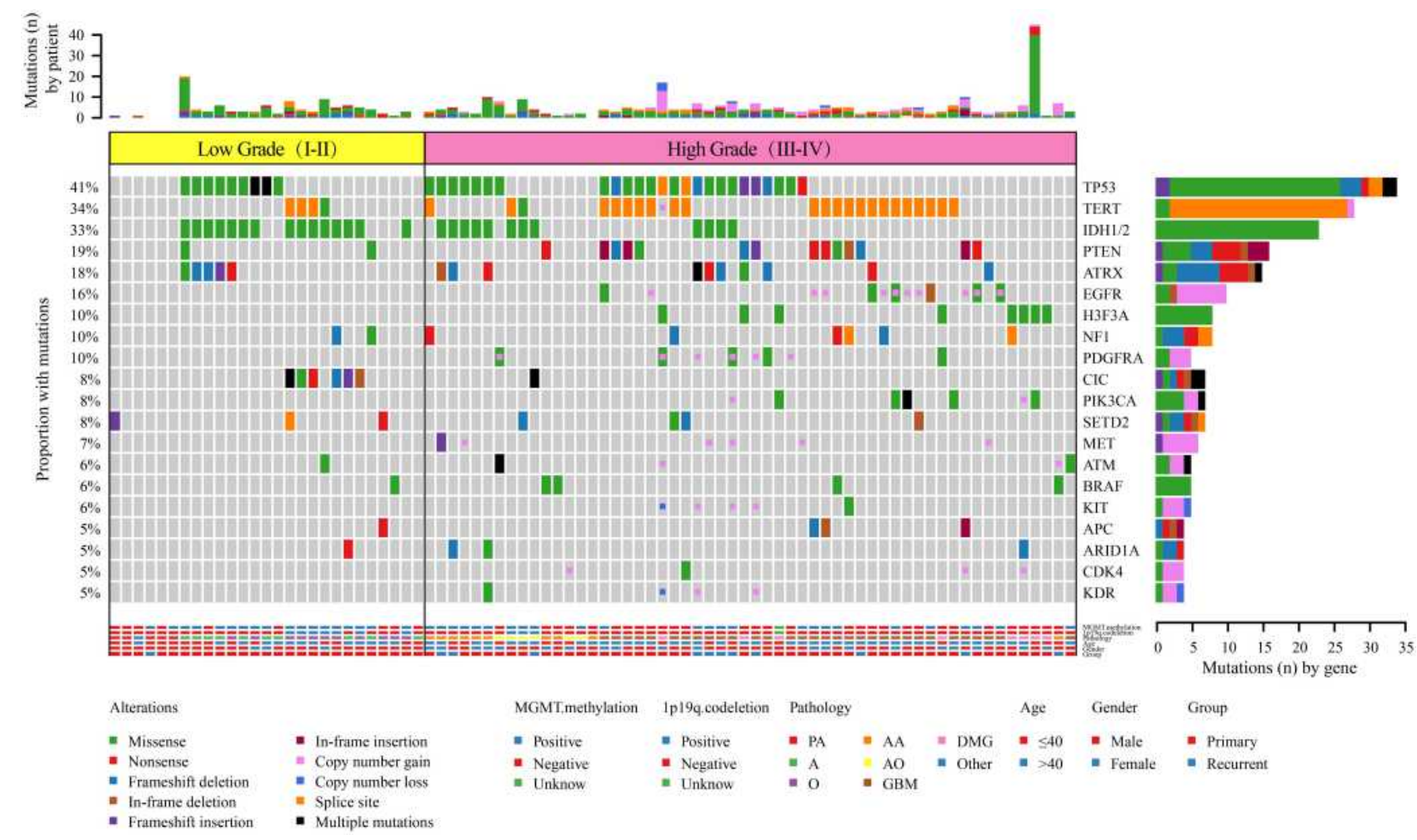

Figure 6 Comparison of the mutational landscape between low-grade (I-II) and high-grade (III-IV) glioma. 
Table 3 Comparison of Important Genes with Somatic Alterations and Clinical Characteristics in Low-Grade (I-II) and High-Grade (III-IV) of Glioma

\begin{tabular}{|c|c|c|c|}
\hline Variable & $I-I I(N=27)$ & III-IV (N=56) & $\mathbf{P}$ \\
\hline TP53 & 9 & 25 & 0.3263 \\
\hline TERT & 4 & 24 & 0.0398 \\
\hline $\mathrm{IDHI} / 2$ & 15 & 12 & 0.0019 \\
\hline PTEN & 2 & 14 & 0.057 \\
\hline EGFR & 0 & 13 & 0.0064 \\
\hline ATRX & 5 & 10 & 0.9415 \\
\hline H3F3A & 0 & 8 & 0.0388 \\
\hline NFI & 2 & 6 & 0.6325 \\
\hline PDGFRA & 0 & 8 & 0.0388 \\
\hline $\mathrm{CIC}$ & 6 & I & 0.0017 \\
\hline PIK $3 C A$ & 0 & 7 & 0.0549 \\
\hline SETD2 & 3 & 4 & 0.3715 \\
\hline MET & 0 & 6 & 0.0774 \\
\hline ATM & I & 4 & 0.5373 \\
\hline$B R A F$ & I & 4 & 0.5373 \\
\hline KIT & 0 & 5 & 0.1092 \\
\hline$A P C$ & I & 3 & 0.7418 \\
\hline ARIDIA & I & 3 & 0.7418 \\
\hline CDK4 & 0 & 4 & 0.1546 \\
\hline$K D R$ & 0 & 4 & 0.1546 \\
\hline$B R C A I$ & 0 & 3 & 0.2206 \\
\hline CDKN2A & 0 & 3 & 0.2206 \\
\hline EPCAM & 0 & 3 & 0.2206 \\
\hline FGFRI & 0 & 3 & 0.2206 \\
\hline FUBPI & 2 & I & 0.1986 \\
\hline $\mathrm{NOTCHI}$ & I & 3 & 0.7418 \\
\hline MGMT & 18 & 25 & 0.0599 \\
\hline$I p \mid 9 q$ & 7 & 3 & 0.0070 \\
\hline $\mathrm{CNV}$ & 0 & 26 & $<0.000$ I \\
\hline Age $\leq 40$ & 14 & 15 & 0.0248 \\
\hline Age $>40$ & 13 & 41 & \\
\hline Male & 24 & 36 & 0.019 \\
\hline Female & 3 & 20 & \\
\hline
\end{tabular}

\section{Discussion}

Improved classification of gliomas using molecular criteria led to a significant revision of the WHO criteria for brain tumors in 2016. ${ }^{32}$ To date, the neuropathology diagnostic laboratory has performed individual tests for selected biomarkers, such as IDH1, IDH2, ATRX, and TERT, as well as mutations in $H 3 F 3 A$ and $B R A F$ and $1 \mathrm{p} / 19 \mathrm{q}$ chromosome deletion. ${ }^{33}$ This involves a variety of detection methods, including the use of mutation-specific antibodies, such as IHC against IDH1-R132H, BRAF-V6OOE, and H3K28M, ${ }^{34}$ conventional Sanger sequencing or tumor DNA pyrosequencing to detect mutations/methylation, and fluorescence or chromogenic in situ hybridization (FISH/CISH) and microsatellite analysis to detect $1 \mathrm{p} / 19 \mathrm{q}$ chromosome deletion. ${ }^{35}$ However, emerging genetic mutations have broad prospects for screening, prognosis, and use as biomarkers. ${ }^{36}$ Recent studies have shown that these standards can be achieved using NGS-based methods. Several studies have found that NGS panels are able to detect known genetic mutations identified by traditional techniques. ${ }^{37,38}$ Our multigene NGS panel simultaneously evaluated multiple mutations, insertions, gene rearrangements, and CNVs associated with gliomas in Chinese patients. Our molecular analysis confirmed that the significant value of NGS targeting gliomas could help improve the classification of brain tumors and could help physicians choose the best targeted therapy. ${ }^{39}$ However, our detection panel was limited in that MGMTmethylation detection was not included in the detection range and must be tested separately using other methods.

A clear distinction among glioma subgroups remains insufficient and needs to be improved to dissect these neoplasms into meaningful biological subgroups. For example, it is unclear how to distinguish between highrisk and low-risk patients with LGG. ${ }^{40}$ Diffuse gliomas comprise most brain tumors, including $\mathrm{A} / \mathrm{AA}$ and $\mathrm{O} / \mathrm{AO} .{ }^{41}$ Their correct pathologic classification could absolutely convey a better outcome in response to targeted therapy. However, diagnosis of these tumors remains challenging because they cannot be confirmed simply based on histological findings, and there is a need for reliable genetic and immunohistochemical markers. ${ }^{42}$ In contrast, targeted NGS or massive parallel sequencing allows accurate examination of a wide range of genetic alterations in tumors simultaneously within a short time. ${ }^{43}$ According to the 2016 WHO CNS tumor classification, targeted NGS used for molecular diagnosis has been routinely applied for diagnosis of these brain tumors. ${ }^{37,44}$ Indeed, numerous studies have reported the analytic value of targeted NGS for routine brain tumor diagnostics. Zacher et al used 20 gene panels to conduct a comprehensive histological and molecular classification of 111 diffuse gliomas, reclassifying OA and GBM based on the status of $I D H$ mutations and identifying tumors with $H 3 F 3 A$ mutations. ${ }^{45}$ Ballester et al used a broader NGS panel (46-50 genes) in 381 brain tumors and found that the marker genes most relevant to brain tumor classification were IDH1/2, TP53, PIK3CA, $B R A F, E G F R, P D G F R A$, and FGFR1/2/3. ${ }^{46}$ Indeed, in our study, 17 samples contained oligodendrocyte components 
A

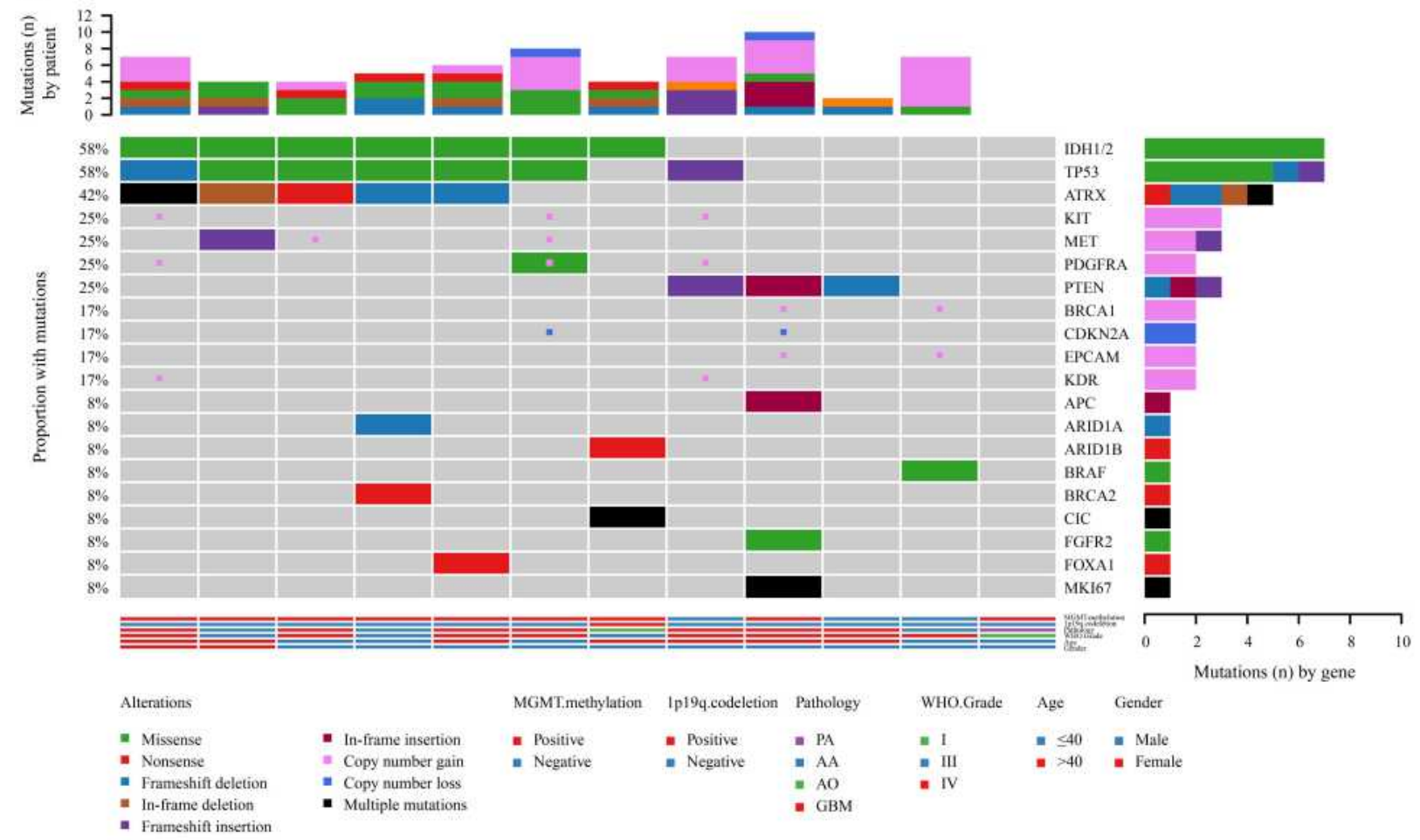

B

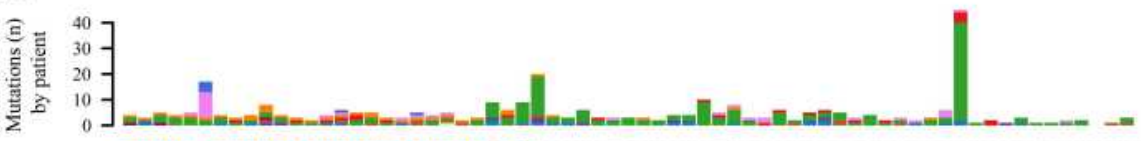

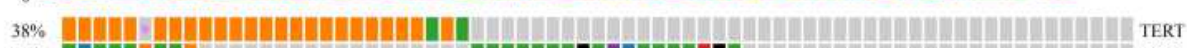
$38 \%$ |

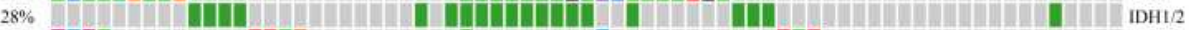

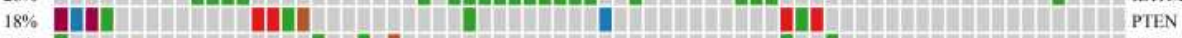

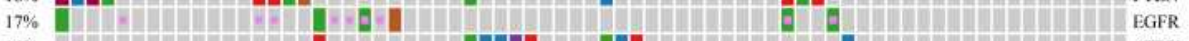

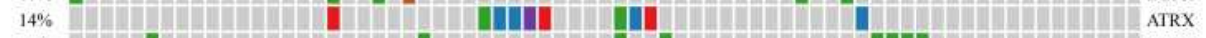

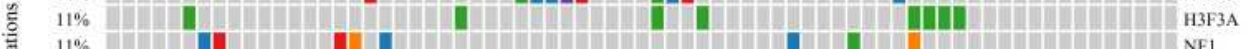
\begin{tabular}{l|l|l|l|l|l|} 
& NF1 \\
$11 \%$ & SETD2
\end{tabular}

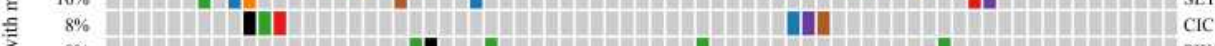

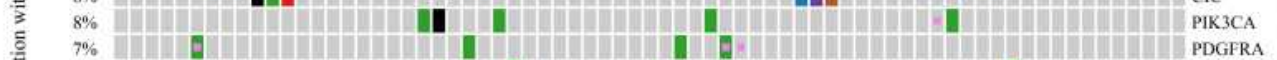
7\% \begin{tabular}{ll|l|l|l|l|l|l|l|l|} 
ATM \\
BRAF
\end{tabular} 4\%

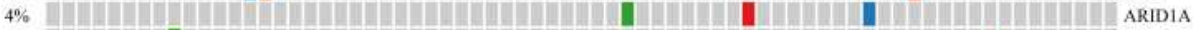
4\% $4 \%$ -

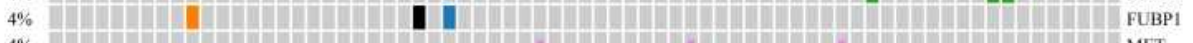
4\%

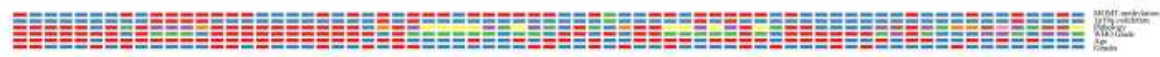

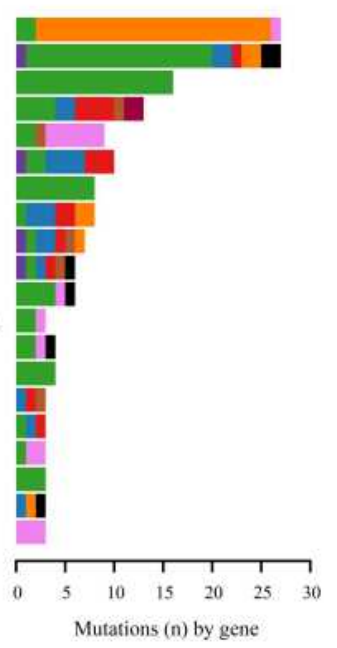

\begin{tabular}{|c|c|c|c|c|c|c|c|c|c|}
\hline Alterations & & MGMTmethylation & Ip19q.codeletion & Pathology & & & WHO.Grade & Age & Gender \\
\hline - Missense & \# In-frame insertion & - Positive & - Positive & $=\mathrm{PA}$ & $=\mathrm{AA}$ & . DMG & $=1$ & n $\leq 40$ & I Male \\
\hline - Nonsense & " Copy number gain & a Negative & m Negative & $A$ & $=\mathrm{AO}$ & $=$ Other & " II & a $>40$ & \# Female \\
\hline - Frameshift deletion & = Splice site & = Unknow & in Unknow & $=0$ & - GBM & & III & & \\
\hline In-frame deletion & - Multiple mutations & & & & & & $=\mathrm{IV}$ & & \\
\hline
\end{tabular}

Figure 7 Mutational landscape of (A) primary gliomas and (B) recurrent gliomas. 
Table 4 Overview of the Distinctive Characteristics for Primary and Recurrent Glioma

\begin{tabular}{|c|c|c|c|}
\hline Variable & Recurrence $(\mathrm{N}=\mid 2)$ & Primary $(\mathrm{N}=7 \mathrm{I})$ & $\mathbf{P}$ \\
\hline TP53 & 7 & 27 & 0.2161 \\
\hline TERT & 1 & 27 & 0.0522 \\
\hline IDHI/2 & 7 & 20 & 0.0391 \\
\hline PTEN & 3 & 13 & 0.6927 \\
\hline EGFR & 1 & 12 & 0.6805 \\
\hline ATRX & 5 & 10 & 0.0365 \\
\hline H3F3A & 0 & 8 & 0.5954 \\
\hline$N F I$ & 0 & 8 & 0.5954 \\
\hline PDGFRA & 3 & 5 & 0.086 \\
\hline $\mathrm{CIC}$ & 7 & 0 & $<0.0001$ \\
\hline PIK3CA & 1 & 6 & $>0.9999$ \\
\hline SETD2 & 0 & 7 & $0.586 \mathrm{I}$ \\
\hline MET & 3 & 3 & 0.0367 \\
\hline ATM & 1 & 4 & 0.5516 \\
\hline BRAF & 1 & 4 & 0.5516 \\
\hline KIT & 3 & 2 & 0.0201 \\
\hline$A P C$ & 1 & 3 & $0.47 \mid 3$ \\
\hline$A R I D I A$ & 1 & 3 & 0.4713 \\
\hline CDK4 & 0 & 4 & $>0.9999$ \\
\hline$K D R$ & 2 & 2 & 0.098 \\
\hline$B R C A I$ & 2 & I & 0.0534 \\
\hline CDKN2A & 2 & 1 & 0.0534 \\
\hline EPCAM & 2 & I & 0.0534 \\
\hline FGFRI & 0 & 3 & $>0.9999$ \\
\hline FUBPI & 0 & 3 & $>0.9999$ \\
\hline NOTCHI & 1 & 2 & 0.3779 \\
\hline MGMT & 9 & 34 & 0.1193 \\
\hline$I p / 9 q$ & 1 & 9 & 0.6691 \\
\hline $\mathrm{CNV}$ & 7 & 19 & 0.0431 \\
\hline Age $\leq 40$ & 5 & 24 & 0.7448 \\
\hline Age $>40$ & 7 & 47 & \\
\hline Male & 10 & 50 & 0.4957 \\
\hline Female & 2 & 21 & \\
\hline WHO IV & 8 & 33 & 0.2268 \\
\hline WHO I-III & 4 & 38 & \\
\hline $\mathrm{A} / \mathrm{O}$ & 0 & 21 & 0.0997 \\
\hline $\mathrm{AA} / \mathrm{AO}$ & 4 & 12 & \\
\hline GBM & 7 & 25 & \\
\hline DMG & 0 & 8 & \\
\hline Others & 1 & 5 & \\
\hline
\end{tabular}

and were originally diagnosed as Grade II-III O/AO. In the subgroup, IDH1/2 mutation (12/17, 71\%), TERT mutation $(6 / 17,35 \%)$, and $1 \mathrm{p} 19 \mathrm{q}$ codeletion $(10 / 17,59 \%)$ were the major genetic alterations. Except for mutations in IDH1/2 and TERT, O/AO also had CIC and FUBP1 mutations. Seven patients did not conform to the $2016 \mathrm{WHO}$
CNS classification criteria of $I D H$ mutated and $1 \mathrm{p} 19 \mathrm{q}$ codeletion. We re-analyzed a series of $\mathrm{AO} / \mathrm{O}$ cases and using an 808-panel NGS assay, refined or reclassified the diagnoses based on the molecular-rich criteria (eg, $B R A F$, $F G F R$ ) provided in the cIMPACT-NOW update4(2) CNS tumor classification. The pathological of these patients have been redefined. In addition, Sahm et al found that their NGS panel identified diagnostic markers and actionable targets for brain tumors. ${ }^{47}$ However, none of these studies further improved the diagnostic value due to a lack of large NGS panel data. In contrast, our study found not only similar genetic markers in brain tumors but also additional and novel mutational and genetic alterations used for the classification and prognosis of brain tumors, such as mutations in $C I C, F U B P 1, T P 53, B R A F, P I K 3 C A$, NF1, FGFR, NOTCH1, EGFR, ATM, and PDGFRA amplification. These novel and clinically actionable variants could assist in exploring better-targeted therapies for glioma treatment.

In this study, the most common mutations in invasive glioma were TP53, TERT, IDH1, PTEN, ATRX, and $E G F R$, consistent with other reports. ${ }^{48,49}$ In astrocytoma grade II/III, 10/11 (91\%) IDH mutation cases also exhibited TP53 mutations, and 4/4 (100\%) IDH1 mutations in GBM also contained TP53 mutations. Neither grade II/III astrocytoma nor grade IV GBM with IDHI mutation exhibited EGFR mutations or PDGFRA amplification. All LGGs and HGGs with gene amplification evidence were primarily $I D H$ wild type, particularly GBM, which was $100 \% I D H$ wild type and exhibited gene amplification. Only $2 \mathrm{IDH}$ mutant gliomas had $M E T$ amplification comutations, indicating that $I D H 1 / 2$ and copy numbers rarely cooccur. $I D H 2$ mutations are rare in GBM. These data are consistent with the existence of distinct genetic pathways for primary (IDH-WT) and secondary (IDHmutant) GBM. ${ }^{50}$

Our estimated gene amplification events based on NGS coverage data were $0 \% \mathrm{O}$ (grade II), 10\% A/AA (grade II/ III), $11.76 \%$ AO tumors (grade III), and 56.25\% GBM (grade IV), indicating that copy number variation is more frequent in high-grade gliomas. EGFR and PDGFR amplification events can be detected by NGS analysis and may guide therapeutic intervention. ${ }^{23}$ For example, clinical trials (NCT01257594, NCT02331693, NCT02101905, and NCT02233049) evaluating EGFR or PDGFR inhibitors, such as erlotinib, lapatinib, and nilotinib, or immunotherapy-based methods for EGFR-overexpressing tumors, as well as regorafenib in relapsed glioblastoma 
(REGOMA) (NCT02926222) ${ }^{51}$ (https://clinicaltrials.gov/), are ongoing. However, before using gene amplification results for clinical decision-making, it is best to use other molecular analyses for proper clinical validation to guide targeted therapy in glioblastoma. ${ }^{52,53}$

Herein, we found that the frequency of CNV events in high-grade gliomas was significantly higher than in low-grade tumors, and the recurrence group had significantly more CNV events than the initial treatment group. Previous studies have found that the existence of EGFR amplification in CNV analysis was significantly associated with both worse disease-free survival (DFS) and overall survival (OS) in gliomas. ${ }^{28,29}$ Obviously, DNA copy number variations, especially amplification on chromosome 7, including EGFR/MET/CDK6, and chromosome 4, including PDGFRA, are commonly observed in gliomas. ${ }^{23} I D H$ mutation is a well-known prognostic factor. According to previous reports, the CNV pattern in $I D H$-mutated gliomas is distinct from that in $I D H$ wildtype groups, and the prognosis is worse. ${ }^{28}$ In comparing our cohort to the MSKCC cohort, which has a primarily Caucasian racial background, some gene mutations, especially the incidence of CNVs, were different. The reason may be differences in ethnicity and clinical characteristics between the two cohorts. In addition, we noted that $C D K N 2 A$ has a lower mutation frequency in Chinese patients. Previous studies have shown that in terms of OS, loss of $C D K N 2 A$ is associated with poor treatment response and shorter OS in diffuse glioma. ${ }^{54}$ However, MET, EPCAM, and ERBB2 CNVs have a higher mutation frequency in Chinese patients, indicating that it is reasonable and feasible to identify risk stratification factors from recurring characteristics.

\section{Conclusions}

Routine use of multigene NGS in the simultaneous evaluation of multiple relevant markers is a reliable and effective method for identifying novel genetic alterations for better classification of brain tumors. This allows clinicians to evaluate prognosis and additional potential therapeutic options, such as targeted therapy, for patients with gliomas according to different racial or ethnic groups. Our findings implicate gene panel NGS as a promising diagnostic technique that may facilitate integrated histological and molecular glioma classification. It is expected that NGS-based molecular analysis may play an increasingly important role in formal cancer classification and treatment of brain tumors in the future.

\section{Ethical Statement}

The study was conducted in accordance with the Declaration of Helsinki (as revised in 2013). This study was approved by the Medical Ethics Committee of Peking University International Hospital (YN2020QN03), and the written informed consent was obtained from each patient. We confirmed all the data used in this study was anonymized or maintained with confidentiality.

\section{Acknowledgments}

The authors wish to thank all patients who participated in this study.

\section{Author Contributions}

All authors made substantial contributions to conception and design, acquisition of data, or analysis and interpretation of data; took part in drafting the article or revising it critically for important intellectual content; agreed to submit to the current journal; gave final approval of the version to be published; and agree to be accountable for all aspects of this work.

\section{Funding}

This work was supported by the Peking University International Hospital Research Grant (No. YN2020QN03).

\section{Disclosure}

Mingwei Li, Huina Wang, Feng Lou, and Shanbo Cao are from Acornmed Biotechnology Co., Ltd. Dr Changyu Lu report non-financial support, other from Acornmed Biotechnology Co., Ltd, during the conduct of the study. All other authors have declared no conflicts of interest.

\section{References}

1. Ostrom QT, Gittleman H, Fulop J, et al. CBTRUS statistical report: primary brain and central nervous system tumors diagnosed in the United States in 2008-2012. Neuro Oncol. 2015;17(Suppl 4):iv1iv62. doi:10.1093/neuonc/nov189

2. Onizuka H, Masui K, Komori T. Diffuse gliomas to date and beyond 2016 WHO classification of tumours of the central nervous system. Int J Clin Oncol. 2020;25(6):997-1003. doi:10.1007/s10147-02001695-w

3. Louis DN, Perry A, Reifenberger G, et al. The 2016 world health organization classification of tumors of the central nervous system: a summary. Acta Neuropathol. 2016;131(6):803-820. doi:10.1007/ s00401-016-1545-1

4. Akagi Y, Yoshimoto K, Hata N, et al. Reclassification of 400 consecutive glioma cases based on the revised 2016WHO classification. Brain Tumor Pathol. 2018;35(2):81-89. doi:10.1007/s10014-0180313-4 
5. Ebrahimi A, Skardelly M, Bonzheim I, et al. ATRX immunostaining predicts IDH and H3F3A status in gliomas. Acta Neuropathol Commun. 2016;4(1):60. doi:10.1186/s40478-016-0331-6

6. Zheng L, Zhang M, Hou J, et al. High-grade gliomas with isocitrate dehydrogenase wild-type and $1 \mathrm{p} / 19 \mathrm{q}$ codeleted: atypical molecular phenotype and current challenges in molecular diagnosis. Neuropathology. 2020;40(6):599-605. doi:10.1111/neup.12672

7. Ghidini M, Petrelli F, Hahne JC, et al. Clinical outcome and molecular characterization of brain metastases from esophageal and gastric cancer: a systematic review. Med Oncol. 2017;34(4):62. doi:10.1007/s12032-017-0919-0

8. Ducray F, Idbaih A, Wang XW, et al. Predictive and prognostic factors for gliomas. Expert Rev Anticancer Ther. 2011;11 (5):781-789. doi:10.1586/era.10.202

9. Cho HJ, Zhao J, Jung SW, et al. Distinct genomic profile and specific targeted drug responses in adult cerebellar glioblastoma. Neuro Oncol. 2019;21(1):47-58. doi:10.1093/neuonc/noy123

10. Appin CL, Brat DJ. Biomarker-driven diagnosis of diffuse gliomas. Mol Aspects Med. 2015;45:87-96. doi:10.1016/j.mam.2015.05.002

11. Cantero D, Mollejo M, Sepulveda JM, et al. TP53, ATRX alterations, and low tumor mutation load feature IDH-wildtype giant cell glioblastoma despite exceptional ultra-mutated tumors. Neurooncol Adv. 2020;2(1):vdz059. doi:10.1093/noajnl/vdz059

12. Barritault M, Meyronet D, Ducray F. Molecular classification of adult gliomas: recent advances and future perspectives. Curr Opin Oncol. 2018;30(6):375-382. doi:10.1097/CCO.0000000000000482

13. Parker NR, Khong P, Parkinson JF, et al. Molecular heterogeneity in glioblastoma: potential clinical implications. Front Oncol. 2015;5:55. doi: 10.3389 /fonc. 2015.00055

14. Ene CI, Holland EC. Personalized medicine for gliomas. Surg Neurol Int. 2015;6(Suppl1):S89-95. doi:10.4103/2152-7806.151351

15. Parilla M, Kadri S, Patil SA, et al. Integrating a large next-generation sequencing panel into the clinical diagnosis of gliomas provides a comprehensive platform for classification from FFPE tissue or smear preparations. $J$ Neuropathol Exp Neurol. 2019;78 (3):257-267. doi:10.1093/jnen/nly130

16. Nikiforova MN, Wald AI, Melan MA, et al. Targeted next-generation sequencing panel (GlioSeq) provides comprehensive genetic profiling of central nervous system tumors. Neuro Oncol. 2016;18(3):379-387. doi:10.1093/neuonc/nov289

17. Movassaghi M, Shabihkhani M, Hojat SA, et al. Early experience with formalin-fixed paraffin-embedded (FFPE) based commercial clinical genomic profiling of gliomas-robust and informative with caveats. Exp Mol Pathol. 2017;103(1):87-93. doi:10.1016/j. yexmp.2017.06.006

18. Li J, Lupat R, Amarasinghe $\mathrm{KC}$, et al. CONTRA: copy number analysis for targeted resequencing. Bioinformatics. 2012;28 (10):1307-1313. doi:10.1093/bioinformatics/bts146

19. Boeva V, Popova T, Bleakley K, et al. Control-FREEC: a tool for assessing copy number and allelic content using next-generation sequencing data. Bioinformatics. 2012;28(3):423-425. doi:10.1093/ bioinformatics/btr670

20. Eckel-Passow JE, Lachance DH, Molinaro AM, et al. Glioma groups based on $1 \mathrm{p} / 19 \mathrm{q}$, IDH, and TERT promoter mutations in tumors. $N$ Engl J Med. 2015;372(26):2499-2508. doi:10.1056/ NEJMoa1407279

21. Jonsson P, Lin AL, Young RJ, et al. Genomic correlates of disease progression and treatment response in prospectively characterized gliomas. Clin Cancer Res. 2019;25(18):5537-5547. doi:10.1158/ 1078-0432.Ccr-19-0032

22. Okamoto I, Sakai K, Morita S, et al. Multiplex genomic profiling of non-small cell lung cancers from the LETS Phase III trial of first-line S-1/carboplatin versus paclitaxel/carboplatin: results of a West Japan oncology group study. Oncotarget. 2014;5(8):2293-2304. doi:10.18632/oncotarget.1906
23. Szerlip NJ, Pedraza A, Chakravarty D, et al. Intratumoral heterogeneity of receptor tyrosine kinases EGFR and PDGFRA amplification in glioblastoma defines subpopulations with distinct growth factor response. Proc Natl Acad Sci US A. 2012;109(8):3041-3046. doi:10.1073/pnas.1114033109

24. Munoz-Hidalgo L, San-Miguel T, Megias J, et al. Somatic copy number alterations are associated with EGFR amplification and shortened survival in patients with primary glioblastoma. Neoplasia. 2020;22(1):10-21. doi:10.1016/j.neo.2019.09.001

25. Brito C, Azevedo A, Esteves S, et al. Clinical insights gained by refining the 2016 WHO classification of diffuse gliomas with: EGFR amplification, TERT mutations, PTEN deletion and MGMT methylation. BMC Cancer. 2019;19(1):968. doi:10.1186/s12885019-6177-0

26. Liu B, Morrison CD, Johnson CS, et al. Computational methods for detecting copy number variations in cancer genome using next generation sequencing: principles and challenges. Oncotarget. 2013;4 (11):1868-1881. doi:10.18632/oncotarget.1537

27. Shao X, Lv N, Liao J, et al. Copy number variation is highly correlated with differential gene expression: a pan-cancer study. BMC Med Genet. 2019;20(1):175. doi:10.1186/s12881-019-0909-5

28. Mirchia K, Sathe AA, Walker JM, et al. Total copy number variation as a prognostic factor in adult astrocytoma subtypes. Acta Neuropathol Commun. 2019;7(1):92. doi:10.1186/s40478-019-0746-y

29. Zhang L, Liu Z, Li J, et al. Genomic analysis of primary and recurrent gliomas reveals clinical outcome related molecular features. Sci Rep. 2019;9(1):16058. doi:10.1038/s41598-019-525159

30. Berzero G, Di Stefano AL, Ronchi S, et al. IDH-wildtype lower grade diffuse gliomas: the importance of histological grade and molecular assessment for prognostic stratification. Neuro Oncol. 2020. doi:10.1093/neuonc/noaa258

31. Crespo I, Vital AL, Nieto AB, et al. Detailed characterization of alterations of chromosomes 7, 9, and 10 in glioblastomas as assessed by single-nucleotide polymorphism arrays. J Mol Diagn. 2011;13 (6):634-647. doi:10.1016/j.jmoldx.2011.06.003

32. Blumcke I, Aronica E, Becker A, et al. Low-grade epilepsy-associated neuroepithelial tumours - the 2016 WHO classification. Nat Rev Neurol. 2016;12(12):732-740. doi:10.1038/ nrneurol.2016.173

33. Reifenberger G, Wirsching HG, Knobbe-Thomsen CB, et al. Advances in the molecular genetics of gliomas - implications for classification and therapy. Nat Rev Clin Oncol. 2017;14(7):434-452. doi:10.1038/nrclinonc.2016.204

34. Cai J, Zhang C, Zhang W, et al. ATRX, IDH1-R132H and Ki-67 immunohistochemistry as a classification scheme for astrocytic tumors. Oncoscience. 2016;3(7-8):258-265. doi:10.18632/ oncoscience. 317

35. Jha P, Sarkar C, Pathak P, et al. Detection of allelic status of $1 \mathrm{p}$ and $19 q$ by microsatellite-based PCR versus FISH: limitations and advantages in application to patient management. Diagn Mol Pathol. 2011;20(1):40-47. doi:10.1097/PDM.0b013e3181e961e9

36. Silantyev AS, Falzone L, Libra M, et al. Current and future trends on diagnosis and prognosis of glioblastoma: from molecular biology to proteomics. Cells. 2019;8(8):863. doi:10.3390/cells8080863

37. Carter JH, McNulty SN, Cimino PJ, et al. Targeted next-generation sequencing in molecular subtyping of lower-grade diffuse gliomas: application of the world health organization's 2016 revised criteria for central nervous system tumors. J Mol Diagn. 2017;19 (2):328-337. doi:10.1016/j.jmoldx.2016.10.010

38. Cantero D, Rodriguez de Lope A, Moreno de la Presa R, et al. Molecular study of long-term survivors of glioblastoma by gene-targeted next-generation sequencing. $J$ Neuropathol Exp Neurol. 2018;77(8):710-716. doi:10.1093/jnen/nly048 
39. Shin H, Sa JK, Bae JS, et al. Clinical targeted next-generation sequencing panels for detection of somatic variants in gliomas. Cancer Res Treat. 2020;52(1):41-50. doi:10.4143/crt.2019.036

40. Geurts M, van den Bent MJ. On high-risk, low-grade glioma: what distinguishes high from low? Cancer. 2019;125(2):174-176. doi:10.1002/cncr.31834

41. Esparragosa I, Diez-Valle R, Tejada S, et al. Management of diffuse glioma. Presse Med. 2018;47(11-12Pt2):e199-e212. doi:10.1016/j. lpm.2018.04.014

42. Iorgulescu JB, Torre M, Harary M, et al. The misclassification of diffuse gliomas: rates and outcomes. Clin Cancer Res. 2019;25 (8):2656-2663. doi:10.1158/1078-0432.CCR-18-3101

43. Moller RS, Hammer TB, Rubboli G, et al. From next-generation sequencing to targeted treatment of non-acquired epilepsies. Expert Rev Mol Diagn. 2019;19(3):217-228. doi:10.1080/14737159.2019.1573144

44. Na K, Kim HS, Shim HS, et al. Targeted next-generation sequencing panel (TruSight Tumor 170) in diffuse glioma: a single institutional experience of 135 cases. $J$ Neurooncol. 2019;142(3):445-454. doi:10.1007/s11060-019-03114-1

45. Zacher A, Kaulich K, Stepanow S, et al. Molecular diagnostics of gliomas using next generation sequencing of a glioma-tailored gene panel. Brain Pathol. 2017;27(2):146-159. doi:10.1111/bpa.12367

46. Ballester LY, Fuller GN, Powell SZ, et al. Retrospective analysis of molecular and immunohistochemical characterization of 381 primary brain tumors. J Neuropathol Exp Neurol. 2017;76(3):179-188. doi:10.1093/jnen/nlw119

47. Sahm F, Schrimpf D, Jones DT, et al. Next-generation sequencing in routine brain tumor diagnostics enables an integrated diagnosis and identifies actionable targets. Acta Neuropathol. 2016;131 (6):903-910. doi:10.1007/s00401-015-1519-8
48. Vaubel RA, Tian S, Remonde D, et al. Genomic and phenotypic characterization of a broad panel of patient-derived xenografts reflects the diversity of glioblastoma. Clin Cancer Res. 2020;26 (5):1094-1104. doi:10.1158/1078-0432.CCR-19-0909

49. Baldock AL, Yagle K, Born DE, et al. Invasion and proliferation kinetics in enhancing gliomas predict IDH1 mutation status. Neuro Oncol. 2014;16(6):779-786. doi:10.1093/neuonc/nou027

50. Miyata S, Tominaga K, Sakashita E, et al. Comprehensive metabolomic analysis of IDH1(R132H) clinical glioma samples reveals suppression of beta-oxidation due to carnitine deficiency. Sci Rep. 2019;9(1):9787. doi:10.1038/s41598-019-46217-5

51. Lombardi G, De Salvo GL, Brandes AA, et al. Regorafenib compared with lomustine in patients with relapsed glioblastoma (REGOMA): a multicentre, open-label, randomised, controlled, Phase 2 trial. Lancet Oncol. 2019;20(1):110-119. doi:10.1016/S1470-2045(18)30675-2

52. Indraccolo S, De Salvo GL, Verza $M$, et al. Phosphorylated Acetyl-CoA carboxylase is associated with clinical benefit with regorafenib in relapsed glioblastoma: REGOMA trial biomarker analysis. Clin Cancer Res. 2020;26(17):4478-4484. doi:10.1158/ 1078-0432.CCR-19-4055

53. Santangelo A, Rossato M, Lombardi G, et al. A molecular signature associated with prolonged survival in glioblastoma patients treated with regorafenib. Neuro Oncol. 2020. doi:10.1093/neuonc/noaa156

54. Reis GF, Pekmezci M, Hansen HM, et al. CDKN2A loss is associated with shortened overall survival in lower-grade (World Health Organization Grades II-III) astrocytomas. J Neuropathol Exp Neurol. 2015;74(5):442-452. doi:10.1097/NEN.0000000000000188

\section{Publish your work in this journal}

Cancer Management and Research is an international, peer-reviewed open access journal focusing on cancer research and the optimal use of preventative and integrated treatment interventions to achieve improved outcomes, enhanced survival and quality of life for the cancer patient.
The manuscript management system is completely online and includes a very quick and fair peer-review system, which is all easy to use. Visit http://www.dovepress.com/testimonials.php to read real quotes from published authors. 